\title{
Front Matter: Volume 10970
}

, "Front Matter: Volume 10970," Proc. SPIE 10970, Sensors and Smart Structures Technologies for Civil, Mechanical, and Aerospace Systems 2019, 1097001 (4 June 2019); doi: 10.1117/12.2534358

Event: SPIE Smart Structures + Nondestructive Evaluation, 2019, Denver, Colorado, United States 


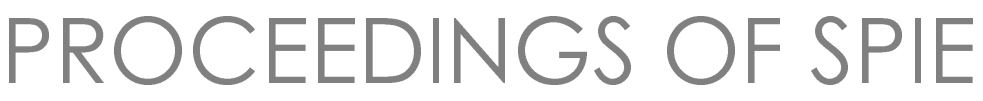

\title{
Sensors and Smart Structures Technologies for Civil, Mechanical, and Aerospace Systems 2019
}

Jerome P. Lynch

Haiying Huang

Hoon Sohn

Kon-Well Wang

Editors

\author{
4-7 March 2019 \\ Denver, Colorado, United States
}

Sponsored by

SPIE

Cosponsored by

OZ Optics, Ltd. (United States)

Polytec, Inc. (United States)

Cooperating Organizations

Jet Propulsion Laboratory (United States)

Colorado Photonics Industry Association (United States)

Published by

SPIE

\section{Volume 10970 \\ Part One of Two Parts}


The papers in this volume were part of the technical conference cited on the cover and title page. Papers were selected and subject to review by the editors and conference program committee. Some conference presentations may not be available for publication. Additional papers and presentation recordings may be available online in the SPIE Digital Library at SPIEDigitallibrary.org.

The papers reflect the work and thoughts of the authors and are published herein as submitted. The publisher is not responsible for the validity of the information or for any outcomes resulting from reliance thereon.

Please use the following format to cite material from these proceedings:

Author(s), "Title of Paper," in Sensors and Smart Structures Technologies for Civil, Mechanical, and Aerospace Systems 2019, edited by Jerome P. Lynch, Haiying Huang, Hoon Sohn, Kon-Well Wang, Proceedings of SPIE Vol. 10970 (SPIE, Bellingham, WA, 2019) Seven-digit Article CID Number.

ISSN: 0277-786X

ISSN: 1996-756X (electronic)

ISBN: 9781510625952

ISBN: 9781510625969 (electronic)

Published by

SPIE

P.O. Box 10, Bellingham, Washington 98227-0010 USA

Telephone +1360 676 3290 (Pacific Time) · Fax +1 3606471445

SPIE.org

Copyright @ 2019, Society of Photo-Optical Instrumentation Engineers.

Copying of material in this book for internal or personal use, or for the internal or personal use of specific clients, beyond the fair use provisions granted by the U.S. Copyright Law is authorized by SPIE subject to payment of copying fees. The Transactional Reporting Service base fee for this volume is $\$ 18.00$ per article (or portion thereof), which should be paid directly to the Copyright Clearance Center (CCC), 222 Rosewood Drive, Danvers, MA 01923. Payment may also be made electronically through CCC Online at copyright.com. Other copying for republication, resale, advertising or promotion, or any form of systematic or multiple reproduction of any material in this book is prohibited except with permission in writing from the publisher. The CCC fee code is $0277-$ $786 \times / 19 / \$ 18.00$.

Printed in the United States of America by Curran Associates, Inc., under license from SPIE.

Publication of record for individual papers is online in the SPIE Digital Library.

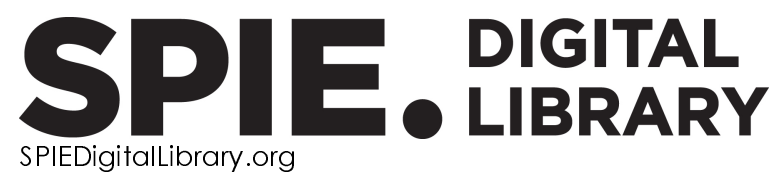

Paper Numbering: Proceedings of SPIE follow an e-First publication model. A unique citation identifier (CID) number is assigned to each article at the time of publication. Utilization of CIDs allows articles to be fully citable as soon as they are published online, and connects the same identifier to all online and print versions of the publication. SPIE uses a seven-digit CID article numbering system structured as follows:

- The first five digits correspond to the SPIE volume number.

- The last two digits indicate publication order within the volume using a Base 36 numbering system employing both numerals and letters. These two-number sets start with $00,01,02,03,04$, 05, 06, 07, 08, 09, OA, OB ... 0Z, followed by 10-1Z, 20-2Z, etc. The CID Number appears on each page of the manuscript. 


\title{
Contents
}

\author{
ix Authors \\ xv Conference Committee
}

\section{Part One}

\section{COMPUTER VISION AND AUGMENTED REALITY SOLUTIONS FOR SHM}

$1097004 \quad$ Non-contact modal parameters identification using a K-cluster algorithm [10970-3]

1097005 An investigation on the relationship between distortions in the motion magnified videos, and the choice of filter bank [10970-4]

1097007 Vision-based precision localization of UAVs for sensor payload placement and pickup for field monitoring applications [10970-7]

\section{ULTRASOUND/GUIDED WAVES}

$1097008 \quad$ Ultrasonic Lamb wave mode conversion to optical fiber guided mode with varying input conditions [10970-8]

1097009 Waveguide sensing for structural health monitoring at elevated temperatures: simulating corrosion damage reconstruction [10970-9]

10970 0A Industrial applications of electro-mechanical impedance technique in novel non-bonded configurations [10970-11]

10970 OB Improved damage isolation using guided waves based on optimized sensor placement [10970-12]

10970 OD Wave focusing in pipe-like structures via gradient-index metamaterial lens toward damage detection and localization [10970-15]

MACHINE LEARNING AND DATA ANALYSIS

10970 OF $\quad$ Addressing sensor drift in a proprioceptive optical foam system [10970-17]

10970 OH DeepSHM: a deep learning approach for structural health monitoring based on guided Lamb wave technique [10970-19] 
10970 ol Prediction of damage location in composite plates using artificial neural network modeling [10970-20]

\section{SMART MATERIALS INTEGRATION FOR SMART SYSTEMS}

10970 OJ SpaceSkin: development of aerospace-grade electronic textile for simultaneous protection and high velocity impact characterization [10970-21]

10970 OK Performance of a water level sensor using magnetostrictive materials [10970-22]

10970 OL A baseline free approach for multiple damage detection in beams [10970-23]

10970 OM An analytical model for a shape memory alloy beam accounting for tension-compression stress asymmetry effect [10970-24]

\section{CASE STUDIES OF SHM IN CIVIL INFRASTRUCTURE SYSTEMS}

10970 ON Sensor data reconstruction and anomaly detection using bidirectional recurrent neural network [10970-25]

$1097000 \quad$ Road vehicle classification using machine learning techniques [10970-26]

10970 OP Reidentification of trucks in highway corridors using convolutional neural networks to link truck weights to bridge responses [10970-27]

$109700 Q \quad$ Instrumentation plan verification for damage detection of a vertical lift steel truss bridge [10970-28]

10970 OR Passive extraction of Green's function of solids and application to high-speed rail inspection [10970-29]

10970 OS Securing critical infrastructures with location based authentication blockchain [10970-30]

10970 OT Large-scale monitoring of retaining structures: new approaches on the safety assessment of retaining structures using mobile mapping [10970-31]

\section{POWER HARVESTING FOR SELF-POWERED SENSORS}

10970 OU Modeling contact electrification in triboelectric impact oscillators as energy harvesters [10970-32]

10970 OW Self-charging and self-monitoring smart civil infrastructure systems: current practice and future trends [10970-34]

10970 OX Vibration-based energy harvesting circuit using feed-forward control [10970-35] 
10970 oY A multistable mechanism to detect thermal limits for structural health monitoring (SHM) [10970-36]

\section{ACTUATORS/ADAPTIVE STRUCTURES}

$109700 Z$ Auto-Gopher-II: an autonomous wireline rotary-hammer ultrasonic drill test results [10970-37]

1097010 Development of synthetic jet actuator array for vortex-flow generation [10970-38]

1097011 Development of a novel actuator for the application of a reconfigurable reflect array antenna [10970-39]

1097012 Aerodynamics and structure measurement subsystem for a shape memory alloy actuated adaptive airfoil [10970-40]

\section{NANOCOMPOSITES AND FLEXIBLE SENSORS}

1097013 On the transient piezoresistive response of impacted nanofiber-modified epoxy [10970-41]

1097014 Stochastic modeling of composite strain and fatigue sensing elements [10970-42]

1097015 Autonomous structural composites for self-powered strain sensing-enabled damage detection [10970-43]

1097016 Weatherability improvement of strain imaging sheet to use in real field for infrastructure inspection technology [10970-44]

$1097017 \quad$ Evaluation of interfacial and micro-damage sensing of composites via Pencil Lead Drawing Paper Sensor (PLDPS) and Electrical Resistance (ER) mapping [10970-45]

1097018 Development of a flexible piezocomposites surface acoustic wave sensor [10970-46]

$1097019 \quad$ Flexible textile antenna sensor for bio-impedance sensing [10970-47]

$109701 \mathrm{~A} \quad$ Experimental identification of stress concentrations in piezoresistive nanocomposites via electrical impedance tomography [10970-48]

PROXIMITY SENSORS FOR IOT SOLUTIONS

10970 1B Detecting anomalies in longitudinal elevation of track geometry using train dynamic responses via a variational autoencoder [10970-49]

10970 1C Incentivizing vehicular crowdsensing system for large scale smart city applications [10970-51]

10970 1D Sleep monitoring using an infrared thermal array sensor [10970-53] 
10970 1E Monitoring induced floor vibrations: dance performance and bridge engineering [10970-55]

$109701 \mathrm{~F} \quad$ Enhancing the imaging performance of electrical capacitance tomography for monitoring osseointegrated prostheses [10970-57]

HEALTH MONITORING OF LARGE-SCALE AND COMPLEX SYSTEMS

$109701 \mathrm{H} \quad$ Online prognosis of fatigue crack at welded joints using nonlinear ultrasonic modulation [10970-50]

$1097011 \quad$ Quantifying the benefit of SHM: can the Vol be negative? [10970-52]

\section{Part Two}

$109701 \mathrm{~J} \quad$ Stress distribution monitoring of ground anchor using optical fiber-embedded strand [10970-54]

$109701 \mathrm{~K} \quad$ Use of bank of Kalman estimators for damage detection of buildings [10970-56]

$109701 \mathrm{~L} \quad$ Advanced sensor for in situ, NDE monitoring of nuclear reactors components integrity [10970-58]

$109701 \mathrm{M} \quad$ Shape memory alloys for earthquake building protection [10970-60]

MODELING OF SMART MATERIALS AND SENSOR PERFORMANCE

$109701 \mathrm{~N} \quad$ Theoretical model for laminated composite beam consisting of multiple superelastic shape memory alloy layers [10970-61]

$109701 Q$ A microscale percolation model for nanocomposite complex impedance [10970-67]

CONTROL AND ACTUATION OF DYNAMIC SYSTEMS

$109701 \mathrm{R} \quad$ Sensor system benefits and costs in positive train control [10970-62]

10970 is Monitoring and control of structures considering diverse uncertainties [10970-64]

$109701 \mathrm{~T} \quad$ Bio-inspired iterative learning technique for more effective control of civil infrastructure [10970-66]

$109701 \mathrm{U}$ Design and analysis of a shock absorber with both tunable inertance and damping [10970-68]

Vi 
OPTICAL FIBER SENSORS

10970 1V Structural health monitoring of solar trackers using distributed fiber optic sensors [10970-69]

$109701 \mathrm{~W}$ Discerning localized thermal impulses using an embedded distributed optical fiber sensor network [10970-71]

$109701 \mathrm{~A} \quad$ Analysis of FBG reflection spectra under uniform and non-uniform transverse loads [10970-73]

$109701 Y \quad$ Test results of lateral load insensitive FBGs embedded in composites to suppress spectral distortion [10970-75]

$1097012 \quad$ Simultaneous position and displacement sensing using two fibre Bragg grating sensors [10970-77]

SHM APPLICATIONS TO CONCRETE STRUCTURES

1097023 Experimental crack detection in concrete pavement using point strain sensors [10970-76]

$1097024 \quad$ Numerical assessment of fatigue life of concrete frame using PZT sensors [10970-78]

SENSOR DEVELOPMENT AND APPLICATIONS

1097026 A high sensitivity piezoelectric MEMS accelerometer based on aerosol deposition method [10970-89]

1097027 CMUT sensors based on circular membranes array for SHM applications [10970-83]

1097028 Analysis of performances of MEMS infrared sensor based on piezoelectric bending resonators [10970-85]

1097029 Application of piezoelectric MFC sensors and fiber Bragg grating sensors in structural health monitoring of composite materials [10970-87]

$109702 \mathrm{~A} \quad \mathrm{CO}_{2}$ sensing characteristics of SAW sensor operated at high temperature [10970-81]

\section{SKIN-BASED DISTRIBUTED SENSING FOR SHM APPLICATIONS}

10970 2C Model-assisted validation of a strain-based dense sensor network [10970-80]

10970 2D Soft-matter pressure sensors for turbulence detection [10970-82] 
$109702 \mathrm{E} \quad$ Large area distributed strain monitoring using patterned nanocomposite sensing meshes [10970-86]

10970 2F Feasibility of force detection in 3D printed flexible material using embedded sensors [10970-88]

$109702 \mathrm{G}$ Sensing sheets based on large area electronics for structural health monitoring of bridges [10970-90]

$109702 \mathrm{H} \quad$ Piezoresistive type graphene nano platelet sensor for SHM application in structural components [10970-92]

\section{POSTER SESSION}

$1097021 \quad$ Robot tracking system research basing on optical sensors [10970-93]

10970 2J Train speed estimation using low-cost GPS receivers [10970-94]

10970 2K Design and analysis of flexible skin based on zero Poisson's ratio hybrid honeycomb [10970-95]

$109702 \mathrm{~L} \quad$ Active self-tuned mass damper for vibration control and continuous monitoring of civil structures [10970-96]

$109702 \mathrm{M} \quad$ Design of a new magneto-rheological pressure seal for rotary shaft [10970-97]

$109702 \mathrm{~N} \quad$ A new magneto-rheological skin for controlling pressure of haptic devices [10970-98]

1097020 Design of a new magneto-rheological damper featuring a hybrid type of piston for lower limb exoskeleton [10970-99]

$109702 \mathrm{P} \quad$ Finite element model updating technique oriented to the bearing capacity improvement of bridges [10970-100]

$109702 \mathrm{Q} \quad 1 \mathrm{MHz}$ high-sensitivity FBG sensor system to measure low energy impact in droplet experiment [10970-101]

$109702 \mathrm{R} \quad$ Optimal design of electrodes for an electrical impedance tomography based flexible sensor [10970-102]

$1097025 \quad$ Fluid-structure interaction analysis for dynamic intraocular pressure monitoring in the human eye [10970-103]

10970 2T Fiber-ring laser sensor system using a fiber Fabry-Pérot filter for ultrasound detection [10970-104]

$109702 \mathrm{U} \quad$ An approach of identifying the parameters of IMFs based on PLF [10970-105]

10970 2W Detecting underground metallic objects of different sizes using synthetic aperture radar 
[10970-107]

$109702 X \quad$ Robot welding seam tracking system research basing on image identify [10970-108]

$109702 Y \quad$ Fabrication of biased-magnetorheological elastomers (B-MRE) based on magnetized ferromagnetic particles [10970-109]

$109702 Z$ Damage detection and localization using random decrement technique on metallic plates [10970-1 10]

1097030 A novel special optical waveguide structure with magneto-optic nonreciprocal phase shift under transversely applied magnetic field [10970-112]

1097031 A new type of electromagnetic system for magnetorheological elastomer (MRE)-based base isolation system [10970-113]

1097032 On-line response and damage estimation of a shear wall structure tested on a shake table using Bayesian filtering [10970-1 14$]$ 
Proc. of SPIE Vol. 10970 1097001-10

Downloaded From: https://www.spiedigitallibrary.org/conference-proceedings-of-spie on 25 Apr 2023 Terms of Use: https://www.spiedigitallibrary.org/terms-of-use 


\section{Authors}

Numbers in the index correspond to the last two digits of the seven-digit citation identifier (CID) article numbering system used in Proceedings of SPIE. The first five digits reflect the volume number. Base 36 numbering is employed for the last two digits and indicates the order of articles within the volume. Numbers start with 00, 01, 02, 03, 04, 05, 06, 07, 08, 09, OA, OB...0Z, followed by 10-12, 20-2Z, etc.

\begin{tabular}{|c|c|}
\hline Abdul Majid, Dayang L., 12 & Chen, Yunquan, 2P \\
\hline Abdullah, Ermira, 12 & Cheng, Junhua, 2T \\
\hline Abidin, Suzana, 12 & Cheng, Lun-Kai, $1 Y, 2 Q$ \\
\hline Achaerandio, A., 1V & Cherston, Juliana, OJ \\
\hline Agüero, Marlon, $1 \mathrm{E}$ & Chia, Leonard, 2J \\
\hline Al-Ansari, Adam M., OY & Choi, Seung-Bok, $2 \mathrm{M}, 2 \mathrm{~N}, 2 \mathrm{O}$ \\
\hline Alavi, Amir H., OW, OY & Chou, Jau-Yu, $1 \mathrm{~K}$ \\
\hline Albright, Tyler B., 14 & Concilio, Antonio, $1 \mathrm{M}$ \\
\hline Alshandah, Mohanad, 23 & Cui, Liang, 30 \\
\hline Al-Tarawneh, Mu'ath, 00 & De Sa, Christopher M., OF \\
\hline Altintepe, Kadir, $1 \mathrm{~L}$ & DeVries, L. K., 17 \\
\hline Alzeyadi, Ahmed, 2W & Do, Xuan Phu, $2 \mathrm{M}, 2 \mathrm{~N}, 2 \mathrm{O}$ \\
\hline Ameduri, Salvatore, $1 \mathrm{M}$ & Dovgalenko, George, $1 \mathrm{~L}$ \\
\hline Anton, Steven R., $2 \mathrm{~F}$ & Du, Xiaosong, $2 \mathrm{C}$ \\
\hline Aono, Kenji, ow & Erazo, Kalil, 32 \\
\hline Arifuku, Michiharu, 16 & Ewald, Vincentius, $\mathrm{OH}$ \\
\hline Atli-Veltin, Bilim, $1 Y$ & Fang, Houfei, 11 \\
\hline Aygun, Levent E., $2 \mathrm{G}$ & Farhangdoust, Saman, Ol \\
\hline Azarbayejani, Mohammad, 29 & Fazzi, Luigi, $1 \mathrm{X}$ \\
\hline Azid, Nuramira, 12 & Ferguson, Max, ON \\
\hline Bäcker, Dennis, 10 & Ferraioli, Massimiliano, $1 \mathrm{M}$ \\
\hline Badescu, Mircea, $\mathrm{OZ}$ & Flynn, Kyle, OK \\
\hline Baek, Y. M., 17 & Fogg, Camille, $1 \mathrm{~T}$ \\
\hline Baghalian, Amin, Ol & Frez, Clifford F., 28 \\
\hline Bai, Xian-Xu, $1 \mathrm{U}$ & Fudouzi, Hiroshi, 16 \\
\hline Balasubramanian, Krishnan, $09,2 \mathrm{H}$ & Gao, Jerry, 23 \\
\hline Bao, Xiaoqi, 0Z, 28 & Garrett, James H., 1B \\
\hline Bar-Cohen, Yoseph, $\mathrm{OZ}$ & Ghosh, Abhishek, 2A \\
\hline Barreiros, Jose A., OF & Ghosh, Sayantan, 09 \\
\hline Batt, Gregory, ou & Gibert, James, OU \\
\hline Benedictus, Rinze, $\mathrm{OH}, 1 \mathrm{Z}$ & Glisic, Branko, $2 \mathrm{G}$ \\
\hline Bergés, Mario, 1B & Gong, Xuewen, 26 \\
\hline Bhalla, Suresh, OA, OL, 24 & Groves, Roger M., OH, 1X, 12 \\
\hline Bhardwaj, Bhavana, 2J & Gupta, Sumit, 1F, 2E \\
\hline Bielak, Jacobo, 1B & Hackney, Drew, 08 \\
\hline Bolognani, Denise, 11 & Hagen, Ronald, $1 Y, 2 Q$ \\
\hline Bourbon, G., 27 & HaO, Zhiliang, $2 R$ \\
\hline Bridgelall, Raj, 1R, 2J & Haq, Moinul, 24 \\
\hline Bussini, Alberto, 2L & Hasni, Hassene, OW, OY \\
\hline Butaud, P., 27 & Hassan, H., 1A \\
\hline Cai, Qinlin, OX & Hayakawa, Michihiro, $1 \mathrm{~J}$ \\
\hline Capriotti, Margherita, OR & Hernandez, J. A., 13 \\
\hline Chakrabartty, Shantanu, OW & Hobeck, Jared D., 14 \\
\hline Chang, Chia-Ming, $1 \mathrm{~K}$ & Hou, Rui, OP \\
\hline Chang, YuSheng, $2 Z$ & Hou, Yangqing, 11 \\
\hline Chen, Zhangjie, 1D & $\mathrm{Hu}, \mathrm{Chao}, 2 \mathrm{C}$ \\
\hline Chen, An, 2C & $\mathrm{Hu}, \mathrm{Jie}, 2 \mathrm{~W}$ \\
\hline Chen, Chao-Ting, 26 & Huang, Haiying, 19 \\
\hline Chen, Xinlei, $1 \mathrm{C}$ & Huang, Ying, 00, 1R, 23 \\
\hline
\end{tabular}


Hwang, Yongmoon, 31

Hyakutake, Tsuyoshi, 16

Ikei, Alec, 2D

Imai, Michio, $1 \mathrm{~J}$

Jackson, Shannon, $\mathrm{OZ}$

Jacobs, Rachel, OK

Jansen, Rob, 1Y, 2Q

Jedynska, Aleksandra, $1 Y$

Jenkins, Brian, IW

Jeong, Seongwoon, ON, OP

Jiang, Shuidong, 11

Jiang, Xuhai, 2T

Jiao, Pengcheng, OW, OY

Jiménez, S., 1V

Joe-Wong, Carlee, 1C

Jones, Nicholas J., OK

Joseph, E., 27

Joyce, Peter, $1 \mathrm{~W}$

Judez, A., $1 \mathrm{~V}$

Jung, Gwang-Yong, $2 S$

Jung, Hyung-Jo, 31

Kalenjuk, Slaven, OT

Kaliske, M., $1 \mathrm{~S}$

Kang, Byung-Hyuk, 2M, 2N, $2 \mathrm{O}$

Kim, Daewon, 18

Kim, Daniel, OZ

Kim, Gi-Woo, $2 S$

Kim, J. H., 17

Kim, Yooil, $2 S$

King, Bryan, $1 R$

Kong, Adam, 1W

Kottaram Amrithanath, Abhishek K., 30

Krishnaswamy, Sridhar, 30

Kudela, Pawel, OB

Kumar, Vivek, $2 \mathrm{G}$

Kye, Seung-Kyung, 31

Laflamme, Simon, 2C

Lajnef, Nizar, OW, OY

Lanza di Scalea, Francesco, OR

Law, Kincho H., ON, OP

Le Moal, P., 27

Le, Tran Huy Thang, $2 \mathrm{~N}$

Lee, Choonghan, $2 Y$

Lee, Hyeong Jae, $0 z$

Lee, Junghoon, 31

Leifsson, Leifur, $2 \mathrm{C}$

$\mathrm{Li}$, Jingke, 2T

Li, Rui, 2R

Liang, Albert, OR

Liao, Wei-Hsin, 26

Lienhart, Werner, OT

Lim, Hyung Jin, $1 \mathrm{H}$

Lipowski, Mathias, 10

Liu, Jingxiao, 1B

Liu, Yang, $2 P$

Loayssa, A., 1V

Loh, Kenneth J., 1F, 2E

López Amo, M., $1 \mathrm{~V}$

Lu, Kai, OY
LU, Pan, 1R, 23, 2J

Lynch, Jerome P., 07, OP

Ma, Tao, $2 X$

Maharjan, Dilendra, $1 \mathrm{E}$

Mai Bui Quoc, Long, 2M, 20

Majidi, Carmel, 2D

Malinowski, Pawel, OB

Mao, Liming, 2T

Mao, Zhu, 05

Mariñelarena, J., 1V

Marte, Roman, OT

Martinez, Selene, 1E

Matheson, Caleb, 29

Mehrabi, Armin, Ol

Mehrkash, Milad, $O Q$

Mellerowicz, Boleslaw, $\mathrm{OZ}$

Metz, Brandon, $\mathrm{OZ}$

Miah, M. S., 15

Milazzo, Alberto, 1X

Misla, Aaron, 15

Mohd. Rafie, Azmin S., 12

Molkenboer, Freek, 1Y, 2Q

Mompó, J. J., $1 \mathrm{~V}$

Mongare, Alfred, 15

Moreu, Fernando, $1 \mathrm{E}$

$\mathrm{MU}$, Wenjun, 2R

Nagarajaiah, Satish, 32

Nakave, Shinji, $1 \mathrm{~J}$

Naqvi, Tabassum, 24

Nasrollahi, Amir, 04

Nazeer, Nakash, 17

Nelson, Charles, 1W

Netchaev, Anton, 2E

Nitta, Hiroyuki, 16

Noble, Michael, OS

Noh, Hae Young, 1B, 1C

Nuzzo, Domenico, 1M

Oh, Jung-Sik, 2S

Ohya, Takao, 16

Oikawa, Masashi, $1 \mathrm{~J}$

Okubo, Kazumasa, $1 \mathrm{~J}$

Okudan, G., OD

Ozevin, D., OD

Özütemiz, Kadri Buğra, 2D

Paradiso, Joseph A., OJ

Park, H. S., 17

Park, J. M., 17

Park, YoungHo, 15

Paulsen, Gale L., OZ

Peckens, Courtney A., $1 T$

Peters, Kara, 08

$\mathrm{Pi}$, Xidong, $1 \mathrm{C}$

Placet, V., 27

Ponder, Robert I., 2F

Qiu, Tao, 2K

Quigley, John, 11

Rais-Zadeh, Mina, 28

Rajabzadeh, Aydin, 1X

Raju, Jayalakshmi, OA 
Ramachandran, Neha Vedavathi, 18

Ramasso, E., 27

Randhawa, Jashanjeet, OL, 24

Rebhan, Matthias J., OT

Resta, Ferruccio, 2L

Ridgeway, Lucas, 29

Ripamonti, Francesco, $2 \mathrm{~L}$

Rizzo, Piervincenzo, 04

Roberts, Heather R., 2F

Rohde, Charles A., 2D

Rojas-Nastrucci, Eduardo A., 18

Ryu, Donghyeon, 15

Safaei, Mohsen, $2 F$

Sampath, Kaushik, 2D

Santini-Bell, Erin, $\mathrm{OQ}$

Sarrafi, Aral, 05

Scott, Valerie, 28

Sethy, Debadatta, $2 \mathrm{H}$

Shahsavari, Vahid, $O Q$

Shepherd, Robert F., OF

Sherrit, Stewart, 0Z, 28

Shi, Yuan, $2 \mathrm{U}$

Shin, P.S., 17

Shu, Xiaowu, 30

Skilskyj, James, 19

Sogabe, Naoki, $1 \mathrm{~J}$

Sohn, Hoon, 1H

Soman, Rohan, OB

Sternini, Simone, $O R$

Sturm, James C., 2G

Sun, Hongwei, 2l, $2 X$

Talakokula, Visalakshi, OA

Tallman, T. N., 13, 1A, 1Q

Tan, Zhicheng, 2P

Tanaka, Yoshikazu, 16

Tansel, Ibrahim N., Ol

Tao, Chuanyi, 2T

Tao, Hongcheng, OU

Tashakori, Shervin, 0 I

Tet, Ng W., 12

Thakur, Supriya, OA

Tobe, Hayato, $1 \mathrm{~J}$

Todoroki, Shin-ichi, 16

Toet, Peter, $1 Y$

Tol, S., OD

Tolliver, Denver D., 1R, 23, 2J

Torbol, Marco, 04

Tsuchiya, Koichi, 16

Ulibarri-Sanchez, Jordan, 15

Umer, R., 1N

Urricelqui, J., $1 \mathrm{~V}$

Valles, Zachary C., $\mathrm{OZ}$

van der Togt, Oana, 1 Y

van Kempen, Floris, $1 Y$

Van Meerbeek, Ilse M., OF

van Megen, Davy, 2Q

Vella, Gianmarco, 2E

Verdin, B., 27

Verma, Naveen, 2G
Verzobio, Andrea, 11

Viet, N. V., OM, $1 \mathrm{~N}$

Vosteen, Amir, 1Y

Wang, Xiaojie, 2R

Wang, Ya, 1D

Wang, Zhaohong, OS

Wee, Junghyun, 08

Wei, Heming, 30

Wei, Yujie, 1B

Wilson, Robert, OR

Wissman, James, 2D

Wu, Wen-Jong, 26

Wu, Maochuan, $2 X$

Wyckoff, Elijah, 1E

$\mathrm{Xu}, \mathrm{Susu}, 1 \mathrm{C}$

Yan, Jin, 2C

Yim, Woosoon, $2 Y$

Yoo, JinHyeong, OK

Yu, Tzuyang, 2W

Yuan, Fuh-Gwo, $2 Z$

Zacny, Kris, $\mathrm{OZ}$

Zagrai, Andrei, 15

Zaki, W., OM, $1 \mathrm{~N}$

Zekkos, Dimitrios, 07

Zhang, Chen, 2A

Zhang, Dengwei, 30

Zhang, Haifeng, 2A

Zhang, Jing, 2T

Zhang, Pei, 1C

Zhang, Tianjiao, $1 \mathrm{~F}$

Zhao, Chang, 2K

Zhao, Kang, 2l

Zheng, Zhang, $2 \mathrm{X}$

Zheng, Zhangru, 2 I

Zhong, Wei-Min, $1 \mathrm{U}$

Zhou, $\mathrm{HaO}, 07$

Zhou, Li, 2K, 2U

Zhou, Lucas, 1E

Zhu, An-Ding, $1 \mathrm{U}$

Zhu, Peter (Xuan), OR

Zhu, Songye, OX

Zonta, Daniele, 11

Zou, Feng, 21

Zurita, J., 1V 
Proc. of SPIE Vol. 10970 1097001-14

Downloaded From: https://www.spiedigitallibrary.org/conference-proceedings-of-spie on 25 Apr 2023 Terms of Use: https://www.spiedigitallibrary.org/terms-of-use 


\section{Conference Committee}

Symposium Chairs

Tribikram Kundu, The University of Arizona (United States)

Gregory W. Reich, Air Force Research Laboratory (United States)

Symposium Co-chairs

Zoubeida Ounaies, The Pennsylvania State University (United States) Hoon Sohn, KAIST (Korea, Republic of)

Conference Chair

Jerome P. Lynch, University of Michigan (United States)

Conference Co-chairs

Haiying Huang, The University of Texas at Arlington (United States)

Hoon Sohn, KAIST (Korea, Republic of)

Kon-Well Wang, University of Michigan (United States)

Conference Program Committee

Hiroshi Asanuma, Chiba University (Japan)

Chih Chen Chang, Hong Kong University of Science and Technology (Hong Kong, China)

Genda Chen, Missouri University of Science and Technology

(United States)

Alison B. Flatau, University of Maryland, College Park (United States)

Branko Glisic, Princeton University (United States)

Faramarz Gordaninejad, University of Nevada, Reno (United States)

Benjamin L. Grisso, Naval Surface Warfare Center Carderock Div.

(United States)

Ryan L. Harne, The Ohio State University (United States)

Jung-Wuk Hong, KAIST (Korea, Republic of)

Neil A. Hoult, Queen's University (Canada)

Ying Huang, North Dakota State University (United States)

Mohammad Reza Jahanshahi, Purdue University (United States)

Gi-Woo Kim, Inha University (Korea, Republic of)

Jeong-Tae Kim, Pukyong National University (Korea, Republic of)

Simon Laflamme, lowa State University (United States)

Hui Li, Harbin Institute of Technology (China)

Jian Li, The University of Kansas (United States)

Suyi Li, Clemson University (United States) 
Wei-Hsin Liao, The Chinese University of Hong Kong (Hong Kong, China)

Chin-Hsiung Loh, National Taiwan University (Taiwan)

Kenneth J. Loh, University of California, San Diego (United States)

Bryan R. Loyola, Sandia National Laboratories (United States)

Theodore E. Matikas, University of loannina (Greece)

Norbert G. Meyendorf, lowa State University of Science and Technology (United States)

Akira Mita, Keio University (Japan)

Yiqing Ni, The Hong Kong Polytechnic University (Hong Kong, China)

Hae Young Noh, Carnegie Mellon University (United States)

Irving J. Oppenheim, Carnegie Mellon University (United States)

Wieslaw M. Ostachowicz, The Szewalski Institute of Fluid-Flow Machinery (Poland)

Kara J. Peters, North Carolina State University (United States)

Piervincenzo Rizzo, University of Pittsburgh (United States)

Donghyeon Ryu, New Mexico Institute of Mining and Technology (United States)

Liming W. Salvino, Office of Naval Research Global (United States)

Fabio Semperlotti, Purdue University (United States)

Wei Song, The University of Alabama (United States)

Wieslaw J. Staszewski, A GH University of Science and Technology (Poland)

Zhongqing Su, The Hong Kong Polytechnic University (Hong Kong, China)

R. Andrew Swartz, Michigan Technological University (United States)

Tyler N. Tallman, Purdue University (United States)

Jiong Tang, University of Connecticut (United States)

Marco Torbol, Ulsan National Institute of Science and Technology (Korea, Republic of)

Ming L. Wang, Northeastern University (United States)

Xingwei Wang, University of Massachusetts Lowell (United States)

Ya Wang, Texas A\&M University (United States)

Yang Wang, Georgia Institute of Technology (United States)

Rosalind M. Wynne, Villanova University (United States)

Fuh-Gwo Yuan, North Carolina State University (United States)

Daniele Zonta, Università degli Studi di Trento (Italy)

Session Chairs

1 Keynote Session

Jerome P. Lynch, University of Michigan (United States)

Haiying Huang, The University of Texas at Arlington (United States) 
2 Computer Vision and Augmented Reality Solutions for SHM

Marco Torbol, Ulsan National Institute of Science and Technology (Korea, Republic of)

Jerome P. Lynch, University of Michigan (United States)

3 Ultrasound/Guided Waves

Kara J. Peters, North Carolina State University (United States)

Serife Tol, University of Michigan (United States)

4 Machine Learning and Data Analysis

Jerome P. Lynch, University of Michigan (United States)

5 Smart Materials Integration for Smart Systems

Tyler N. Tallman, Purdue University (United States)

Donghyeon Ryu, New Mexico Institute of Mining and Technology (United States)

6 Case Studies of SHM in Civil Infrastructure Systems

Francesco Lanza di Scalea, University of California, San Diego (United States)

Denise Bolognani, University degli Studi di Trento (Italy)

Piervincenzo Rizzo, University of Pittsburgh (United States)

7 Power Harvesting for Self-Powered Sensors

Hae Young Noh, Carnegie Mellon University (United States)

Zhongqing Su, The Hong Kong Polytechnic University (Hong Kong, China)

8 Actuators/Adaptive Structures

Yoseph Bar-Cohen, Jet Propulsion Laboratory (United States)

Ermira Abdullah, University Putra Malaysia (Malaysia)

9 Nanocomposites and Flexible Sensors

Daewon Kim, Embry-Riddle Aeronautical University (United States)

Haiying Huang, The University of Texas at Arlington (United States)

10A Proximity Sensors for loT Solutions

Ya S. Wang, Texas A\&M University (United States)

Kenneth J. Loh, University of California, San Diego (United States)

10B Health Monitoring of Large-Scale and Complex Systems

Hyung Jim Lim, KAIST (Korea, Republic of)

11 A Modeling of Smart Materials and Sensor Performance

Lingyu Yu, The University of South Carolina (United States) 
$11 \mathrm{~B}$ Control and Actuation of Dynamic Systems

Courtney Peckens, Hope College (United States)

Raj Bridgelall, North Dakota State University (United States)

12A Optical Fiber Sensors

R. Brian Jenkins, U.S. Naval Academy (United States)

12B SHM Applications to Concrete Structures

Branko Glisic, Princeton University (United States)

Ying Huang, North Dakota State University (United States)

13A Sensor Development and Applications

Haifeng Zhang, University of North Texas (United States)

Wei-Hsin Liao, The Chinese University of Hong Kong

(Hong Kong, China)

13B Skin-based Distributed Sensing for SHM Applications

Kenneth J. Loh, University of California, San Diego (United States)

Austin Downey, The University of South Carolina (United States) 